\title{
INTERCOMPARAÇÃO ENTRE QUATRO MÉTODOS DE ESTIMATIVA DA ALTURA DA CAMADA LIMITE CONVECTIVA DURANTE O EXPERIMENTO RACCI - LBA (2002) EM RONDÔNIA - AMAZÔNIA
}

\author{
LUIZ ANDRÉ R. DOS SANTOS ${ }^{1}$ E GILBERTO FISCH ${ }^{2}$ \\ ${ }^{1}$ Instituto Nacional de Pesquisas Espaciais (INPE) \\ Avenida dos Astronautas, 1758 - CEP 12227-010, São José dos Campos, SP, Brasil \\ Email: landre@cptec.inpe.br \\ ${ }^{2}$ Centro Técnico Aeroespacial (CTA) \\ Praça Marechal Eduardo Gomes, 50 - CEP 12228-904 São José dos Campos - SP - Brasil \\ Email: gfisch@iae.cta.br
}

Recebido Março 2006 - Aceito Junho 2006

\begin{abstract}
RESUMO
Este trabalho tem como objetivo fazer uma comparação entre quatro diferentes métodos de estimativa da altura da Camada Limite Convectiva (CLC), utilizando dados obtidos pelo equipamento SODAR e por radiossondas, durante a campanha DRY - to - WET do experimento RaCCI - LBA (2002) em Rondônia. Os quatro métodos de estimativa utilizados foram os seguintes: o das Parcelas; do Número de Richardson; dos Perfis; e um método de medição direta através do SODAR. Os resultados mostraram que o Método das Parcelas superestimou as alturas da CLC (em 15\% para às 08:00 HL e 14:00 HL e 30\% nos demais horários), em quase todos os casos analisados, obtendo índices baixos do coeficiente de determinação $\left(\mathbf{r}^{2}\right)$, quando comparado com os outros métodos. O Método do Número de Richardon e o Método dos Perfis apresentaram alturas muito semelhantes, com diferenças médias de menos de $50 \mathrm{~m}$, alcançando um valor de $\mathbf{r}^{2}$ igual a 0,97 , durante o período de início da estação chuvosa. As medidas realizadas com o SODAR não se mostraram adequadas nas primeiras horas da manhã, indicando que o equipamento mediu a Camada Limite Residual (do dia anterior) e não a Camada Limite Convectiva rasa, em fase de desenvolvimento.

Palavras-chave: Número de Richardson, Perfis de temperatura e umidade específica, SODAR.
\end{abstract}

ABSTRACT: INTERCOMPARATION BETWEEN FOUR METHODS OF ESTIMATE THE CONVECTIVE BOUNDARY LAYER HEIGHT DURING THE RaCCI - LBA EXPERIMENT (2002) IN RONDÔNIA - AMAZÔNIA.

This work has like objective to do a comparison between four different methods of estimate of the Convective Boundary Layer (CBL) height, utilizing data obtained by the SODAR and radiossound, during the DRY - to - WET campaign of the RaCCI - LBA experiment in Rondonia, (2002). The four methods of estimate utilized were: the Parcel Method; the Richardson Number Method; the Profiles Method; and a direct measurement method by SODAR. The results showed that the Parcel Method overestimated the heights of the CBL (15\% at 08:00 LT and 14:00 LT, and 30\% to the other hours), in most of cases analyzed, obtaining a short determination index $\left(\mathbf{r}^{2}\right)$, when compared with the others methods. The Richardson Number Method and the Profiles Method presented very similar heights, with average differences less than $50 \mathrm{~m}$, achieving a $\mathbf{r}^{2}$ value about 0,97 , during the beginning of the rainy station. The SODAR data was not adequate at the first hours of the morning, showing that the equipment takes the Residual Boundary Layer (from the previous day) and not the shallow Convective Boundary Layer, in development.

Keywords: Richardson Number, temperature and moisture profile, SODAR. 


\section{INTRODUÇÃO}

O desmatamento de áreas de floresta tropical amazônica, como, por exemplo, para a extração de madeira e a substituição dessas áreas naturais por pastagens e/ou áreas agrícolas, gera efeitos nos climas local, regional e global. Estes efeitos tem sido estudados através de simulações climáticas usando Modelos de Circulação Geral da Atmosfera (MCGA), como o trabalho pioneiro realizado por Nobre et al. (1991). Posteriormente, vários outros estudos foram realizados, com resultados similares. Porém, para que haja um aperfeiçoamento desses modelos e uma conseqüente melhora dos resultados e previsões, é necessário, além de parametrizações dos processos físicos mais adequadas, um entendimento detalhado das interações dos mecanismos dinâmicos e termodinâmicos, e ainda, daqueles associados com a Camada Limite Atmosférica - CLA ( Rocha, 1992 ).

A CLA responde as forçantes da superfície, com uma escala de tempo de aproximadamente 1 hora ou menos e apresenta, também, uma escala de altura da ordem de aproximadamente $1 \mathrm{a} 2 \mathrm{~km}$. Estas forçantes incluem o arrasto friccional, a evaporação e a transpiração, a transferência de calor, a emissão de poluentes e a modificação do escoamento induzido pela superfície (Stull, 1988). Por este motivo faz-se necessário o conhecimento da altura da Camada Limite Convectiva (CLC) para o entendimento da estrutura e dos processos físicos e dinâmicos que ocorrem no interior desta camada, sendo que a altura da CLC é uma das variáveis mais importantes de se conhecer e prever.

Este trabalho tem o objetivo de fazer uma intercomparação entre quatro diferentes métodos de estimativas da altura da CLC, sendo um método subjetivo (Método dos Perfis), dois métodos objetivos (Método do Número de Richardson e Método das Parcelas) e um método de medição direta através de equipamento SODAR.

\section{MATERIAL E MÉTODOS}

Na região de Ji-Paraná - RO encontram-se áreas de floresta e de pastagem, suficientemente grandes para desenvolver seus próprios microclimas, e pelo fato dessas áreas coexistirem próximas umas das outras, a região tem sido alvo de vários projetos e pesquisas que estudam o impacto do desmatamento no clima (Fisch, 1996). Entre o período de 15 de setembro a 30 de outubro de 2002 foi realizada uma campanha de coleta de dados durante a transição entre as estações seca e chuvosa no Sudoeste da Amazônia, pelo projeto denominado RaCCI - Radiation, Cloud, and Climate Interactions in the Amazon during the DRY-TO-WET Transition Season/LBA (Interações entre Radiação, Nuvens e Clima na Amazônia na Transição entre as Estações Seca e Chuvosa). Maiores informações sobre este experimento encontram-se na página do projeto (www.master. iag.usp.br/lba).

O conjunto de dados utilizado neste trabalho foi obtido no sítio de pastagem, localizado na fazenda Nossa Senhora Aparecida, no município de Ouro Preto do Oeste, RO a $10^{\circ}$ $46^{\prime} \mathrm{S}$ de latitude e $62^{\circ} 20^{\prime} \mathrm{W}$ de longitude, com uma altitude de 293 metros acima do nível do mar, na região de Ji-Paraná (RO). O sítio está centrado numa área de $50 \mathrm{~km}$ de raio, quase que totalmente desmatada. Medidas realizadas por satélites mostram que somente $5 \%$ da área de $10 \times 10 \mathrm{~km}$ possuem ainda floresta tropical, sendo o restante de área desmatada para atividades de agropecuária. Maiores detalhes sobre as características fisiográficas em Fisch (1996).

Os dados utilizados para a determinação e comparação das alturas foram coletados em duas fases distintas, sendo a Fase I compreendida entre os dias 18 a 30 de setembro de 2002; e a Fase II compreendida entre 15 a 29 de outubro de 2002. As duas fases foram divididas desta forma por apresentarem características diferentes uma da outra, pois a Fase I se apresenta no final do período seco, com influência de queimadas locais, comuns nessa época do ano, e a Fase II sendo caracterizada por ser o início da estação chuvosa na região (Santos, 2005).

\subsection{Dados}

Para a estimativa das alturas da CLC pelos métodos objetivos e subjetivo foram utilizadas radiossondagens (sonda RS80-15G da Vaissala Oy) que coletam dados de altitude (m), temperatura $\left(\mathrm{C}^{\mathrm{O}}\right)$, umidade específica $\left(\mathrm{g} \mathrm{kg}^{-1}\right)$, pressão $(\mathrm{hPa})$, direção (graus) e velocidade $\left(\mathrm{m} \mathrm{s}^{-1}\right)$. A determinação dos ventos utiliza a técnica do GPS. Maiores detalhes sobre este tipo de medida encontram-se em Fisch et al. (1998). Estas sondagens foram realizadas nos horários das 08:00 Hora Local (HL), 11:00 HL, 14:00 HL e 17:00 HL, que mostram a evolução da CLC.

Os dados de SODAR - Doppler (Sound Detection and Ranging) foram obtidos do modelo Remtech PA2 (Phased Array), também instalado na Fazenda Nossa Senhora. O SODAR é um instrumento monoestático, funciona com $10 \mathrm{~W}$ de potência e freqüência de $2 \mathrm{kHz}$, com uma antena transmissora/receptora de $1,4 \mathrm{~m}^{2}$, onde os pulsos acústicos são emitidos a cada 5 segundos e os dados armazenados com um intervalo de tempo de 30 minutos. O SODAR possui um alcance médio de até $1500 \mathrm{~m}$ de altura. O equipamento, além de obter os perfis vertical direção e velocidade do vento e estrutura de turbulência, foi programado para fornecer a altura da inversão térmica em altitude, assumida como sendo a altura da CLC. Andrade et al. (2004) utilizou este mesmo equipamento para medidas comparativas na região do Pantanal brasileiro e concluiram que o equipamento não consegue representar adequadamente nos horários de máxima atividade convectiva (entre 14:00 e 17:00 HL). 


\subsection{Metodologia}

Foi calculada a altura da $\boldsymbol{C L C}(\mathbf{h})$ através de três métodos diferentes de estimativas desta altura e um método de medida direta, sendo que estes métodos são descritos a seguir:

\section{Método Subjetivo}

Os perfis de temperatura e de umidade específica na CLA são usados para uma estimação subjetiva de $\mathbf{h}$. Sob condições convectivas, esta altura é comumente identificada com a base de uma elevada camada de inversão ou estável, e também como sendo a altura da redução na umidade específica do ar, que geralmente é acompanhada pelo cisalhamento do vento.

O Método dos Perfis é baseado no fato que, dentro da CLC, as propriedades da temperatura potencial $(\theta)$ e umidade específica (q) são bem misturadas, permanecendo quase que constantes com a altura (z) e, ao atingirem a camada de inversão, o gradiente dessas propriedades apresenta uma forte inclinação, deixando assim de ser constantes (Stull, 1988). Ao atingir esta camada estável, determina-se a camada de inversão, sendo que a altura da CLC corresponde à base desta camada no primeiro ponto onde o perfil da temperatura aumenta e o perfil da umidade específica decresce rapidamente, com a altura, por pelo menos três pontos consecutivos (Fisch et al., 2004). O ponto mais baixo é adotado como a altura da CLC. Quando este critério para a

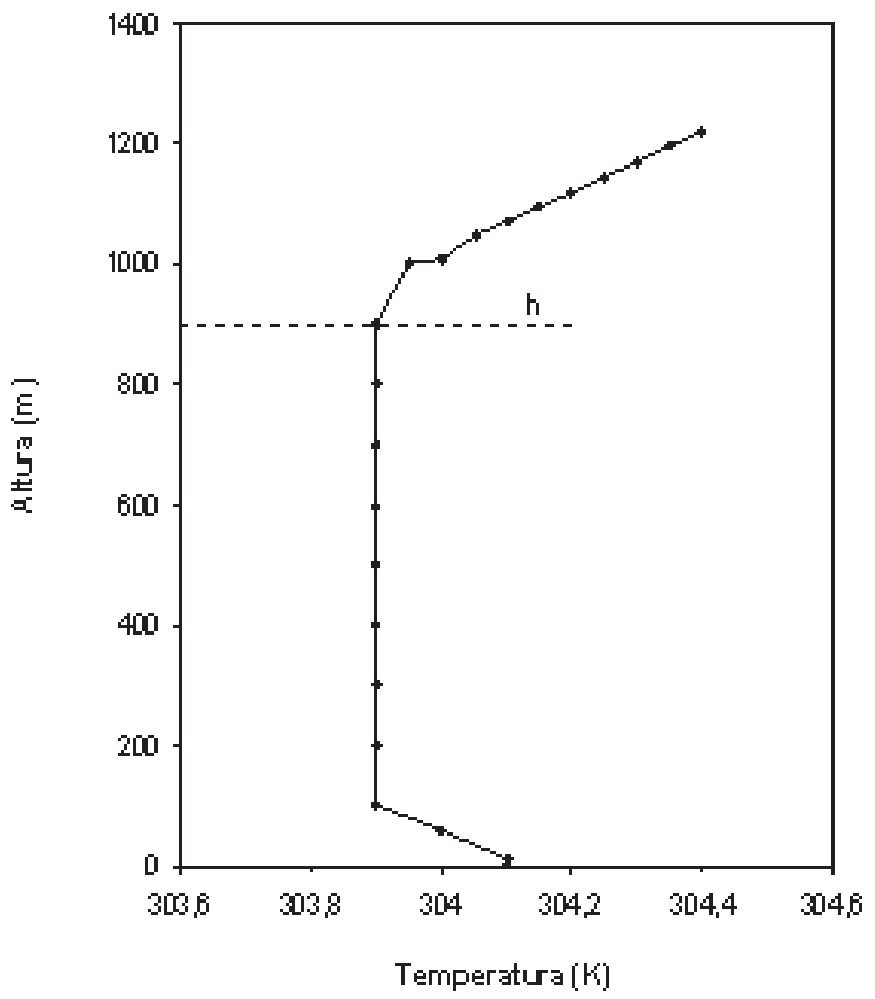

Figura 1 - Representação ideal do Método do Perfil de $\theta$. estimativa da altura da CLC não é estabelecido em ambos os perfis de temperatura potencial e de umidade específica, optase pelo perfil de $\theta$, pois sabe-se que em uma sondagem podem existir mais erros com o sensor de umidade do que com o sensor de temperatura (Santos, 2005). Este método está esquematizado na Figura 1.

\section{Métodos Objetivos}

Alguns métodos objetivos foram desenvolvidos para simplificar e homogeneizar a estimação de $\mathbf{h}$ em condições convectivas. A idéia básica do Método das Parcelas é a de seguir a curva da adiabática seca, começando a partir da superfície com a temperatura média (supostamente temperatura máxima), até a intersecção com o perfil de temperatura do ar, como representado na Figura 2. Isto determina a altura $\mathbf{h}$ como sendo um nível de equilíbrio de um hipotético crescimento de parcelas de ar, representando uma térmica. Seibert et al. (2000) citam que a altura encontrada pelo Método das Parcelas não é fortemente correlacionada com as concentrações de gases traço observadas. Este método tem sido bastante utilizado em meteorologia operacional, pela sua praticidade e simplicidade (Seibert et al., 2000). Observa-se que as Figuras 1 e 2 foram feitas na mesma escala horizontal e vertical, para explorar as diferenças de altura por estes dois métodos distintos.

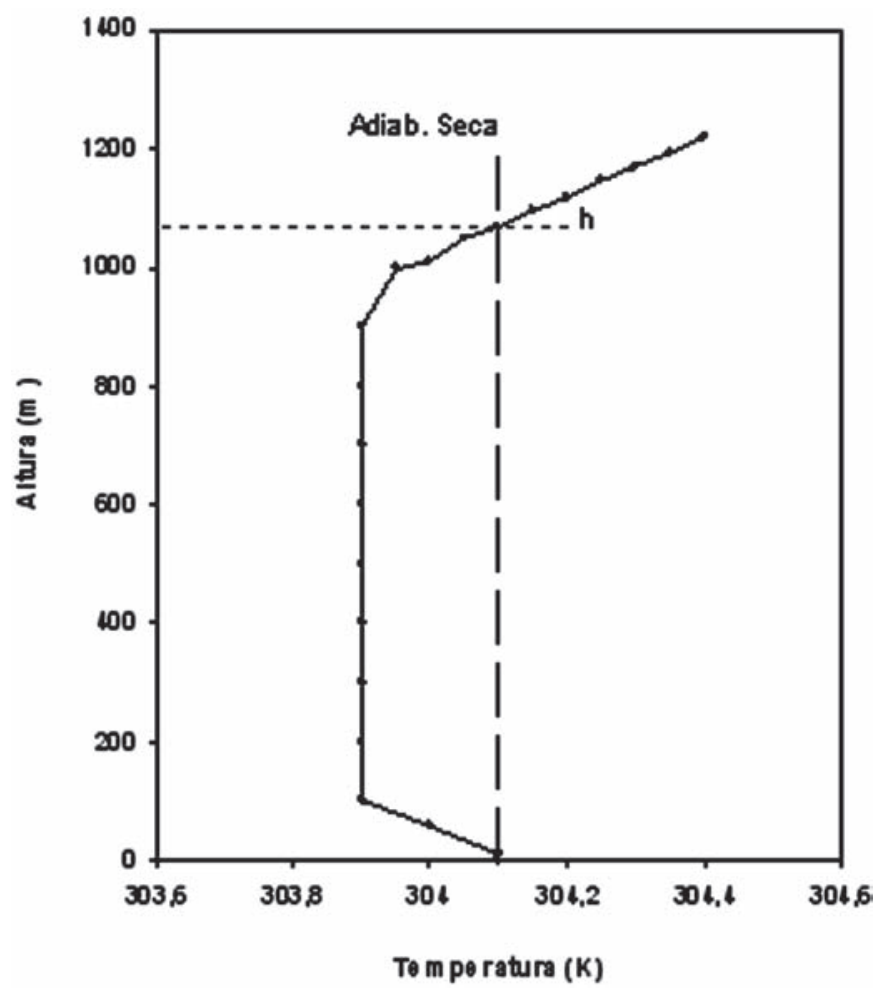

Figura 2 - Representação ideal do Método das Parcelas. 
Uma outra maneira do cálculo de h é utilizando o Método do Número de Richardson (Ri), o qual difere muito na escolha do nível para a temperatura próximo a superfície, a parametrização da produção de cisalhamento pela turbulência na camada superficial e a consideração de uma temperatura excessiva, sob condições convectivas (Vogelezang e Holtslag, 1996).

Para estimar a altura de uma camada limite turbulenta pelo Método do Número de Richardson, utilizou-se a equação proposta por Vogelezang e Holtslag (1996):

$$
\mathrm{R}_{\mathrm{i}}=\frac{\mathrm{g}}{\theta}\left(\frac{\left(\theta_{2}-\theta_{1} / \mathrm{z}_{2}-\mathrm{z}_{1}\right)}{\left(\left(\mathrm{v}_{2}-\mathrm{v}_{1}\right)^{2} /\left(\mathrm{z}_{2}-\mathrm{z}_{1}\right)^{2}\right)}\right)
$$

no qual $\theta$ corresponde a temperatura potencial média da camada $(\mathrm{K}), \theta_{1}$ e $\theta_{2}$ são as temperaturas na base e no topo da camada, $\mathbf{v}_{\mathbf{1}}$ e $\mathbf{v}_{\mathbf{2}}$ é o vento na base e no topo da camada $\left(\mathrm{m} \mathrm{s}^{-1}\right), \mathbf{z}_{\mathbf{1}}$ e $\mathbf{z}_{2}$ é a altura da base e do topo da camada (m) e g é a aceleração da gravidade. No interior da CLC os valores de $\mathrm{R}_{\mathrm{i}}$ devem ser próximos ou igual a zero, sendo que a partir do momento em que estes valores são superiores a um valor crítico (assumido como 0,25 conforme recomendado por Seibert et al., 2000), implica que está havendo uma maior variação nos valores de $\theta$ e dentro da CLC estas variações devem ser muito baixas (0,3 $\mathrm{K}$ ) ou iguais a zero, ou seja, quando os valores de $\mathrm{R}_{\mathrm{i}}$ aumentam demasiadamente, toma-se o ponto como a altura da CLC.

\section{SODAR}

As medidas do SODAR são possíveis devido à emissão de fortes pulsos acústicos na banda de áudio, da superfície para atmosfera, e detectando a freqüência do eco recebido, que varia com a velocidade do vento em várias alturas (efeito Doppler) e a intensidade do eco recebido, que depende da intensidade e estrutura térmica da turbulência atmosférica. Este instrumento é capaz de detectar a altura da camada de inversão através do "método eco", que consiste na associação de $\mathbf{h}$ com a altura do maior gradiente no perfil do eco (Baumann e Piringer, 2001). Beyrich (1995) encontrou resultados coerentes entre a altura da camada de mistura com a altura da máxima intensidade do eco acústico. Porém este método possui a restrição de que a altura da CLC deve estar dentro do alcance vertical máximo do SODAR. Outro método utilizado para a estimativa de $\mathbf{h}$ através do SODAR é a técnica desenvolvida pelo fabricante (método Remtech), que consiste em um "software" e com uma rotina interna para a determinação da altura de mistura baseada na análise espectral da velocidade do vento (Remtech, 1994). A partir deste espectro de velocidade vertical pode ser deduzida uma escala de freqüência característica dos vórtices mais energéticos que, quando multiplicados por uma escala de velocidade do vento, determina a altura da camada de mistura sobre condições convectivas. A vantagem deste método é o fato de se poder estimar as alturas da CLC mesmo que estejam acima do alcance efetivo do SODAR.

\section{RESULTADOS E DISCUSSÃO}

Através dos métodos descrito anteriormente foi possível calcular e comparar a altura da camada limite em quatro horários diferentes que mostram a evolução diurna da CLC, às 08:00 HL, 11:00 HL, 14:00 HL e às 17:00 HL, tanto para a Fase I quanto para a Fase II do experimento.

Para a Fase I (Figura 3) observa-se que o Método das Parcelas, na maior parte dos casos, superestima as medidas obtidas pelos outros três métodos. Em 98 casos de comparações feitas com este método, tem-se que $81 \%$ das alturas encontradas pelo Método das Parcelas foram superiores aos demais. As correlações foram melhores para os Métodos do Número de Richardson e dos Perfis $\left(r^{2}=0,66\right.$ e $r^{2}=0,59$, respectivamente). Excetuandose o caso das comparações realizadas às 08:00 HL com os dados do SODAR (Figuras 3a, 3d e 3e) os outros horários não apresentam um viés sistemático de superestimativas ou subestimativas. Os valores encontrados variam entre 400 e $2400 \mathrm{~m}$. Já para a Fase II, o número de casos analisados foi maior (em torno de 150 pares de valores) e o Método das Parcelas superestimou em cerca de $88 \%$ das alturas encontradas nos outros métodos, sendo que durante os horários das 08:00 HL e 14:00 HL, o Método das Parcelas apresentou alturas aproximadamente $15 \%$ mais altas que os métodos de Richardson e do Perfil e, durante os demais horários, apresentou alturas de cerca de $30 \%$ mais elevadas. Para esta fase os índices de correlação encontrados entre o Método das Parcelas e os outros três foram menores, sendo que o maior índice encontrado foi na comparação com o Método dos Perfis $\left(r^{2}=0,25\right)$, como pode ser visto na Figura $4 \mathrm{c}$. Um motivo para este comportamento pode ser devido ao fato das temperaturas da superfície serem maiores na Fase II do que na Fase I (Santos, 2005), o que resultou em alturas mais elevadas. Hennemuth e Lammert (2006) também sugerem que este método possa ser uma superestimativa, comparando com dados obtidos a partir de LIDAR para diferentes localidades.

O SODAR apresentou as piores comparações, obtendo os menores índices de determinação, tanto na Fase I como na Fase II; além de apresentar valores muito elevados das alturas da CLC às 08:00 HL, chegando até $1145 \mathrm{~m}$, no dia 19/09. Isto pode ser devido ao fato de, uma vez que neste horário a altura média da CLC ainda é baixa, em torno de $400-500 \mathrm{~m}$ (Fisch et al., 2004 e Santos, 2005), os valores encontrados correspondem a altura de inversão da Camada Limite Residual (CLR) proveniente do dia anterior. Os melhores índices do SODAR, porém baixos, foram encontrados na comparação com o Método do Número de Richardson e com Método dos Perfis, com valores iguais a 
0,10 e 0,16 respectivamente (Figuras $3 \mathrm{~d}$ e $3 \mathrm{e}$ ), enquanto que na comparação com o Método das Parcelas este valor foi de 0,04 (Figura 3a). Durante a Fase II, as correlações do SODAR com os outros Métodos foram ainda menores, uma vez que o número de casos comparados entre os métodos aumentou para aproximadamente 50. O maior índice encontrado foi também, na comparação com o Método do Número de Richardson com um valor de $\mathrm{r}$ igual a 0,08 (Figura 4d). Apesar da Fase II ser uma fase onde a CLC apresenta-se mais fria e mais úmida, com alturas da CLC menores que a Fase I (Santos, 2005), os valores encontrados pelo SODAR às 08:00 HL foram muito elevados, caracterizando, assim como na Fase I, valores correspondentes a altura da CLR.

O Método dos Perfis, que é um método utilizado para a determinação da altura da CLC (Seibert et al., 2000), só não obteve bons índices, durante a Fase I, com os valores medi- dos pelo SODAR (Figura 3e), sendo que o valor de 0,59 foi encontrado para a comparação com o Método das Parcelas (Figura 3c) e também obteve o melhor índice entre os métodos, quando comparado com o Método do Número de Richardson, chegando a 0,85 (Figura 3f). Apesar das alturas terem sido bem próximas, nota-se que aquelas calculadas pelo Método do Número de Richardson foram quase sempre menores do que as calculadas pelo Método dos Perfis. Na Fase II houve uma queda nos índices do Método dos Perfis com o método das Parcelas e com as medidas do SODAR, com valores de 0,25 e 0,06, respectivamente (Figuras 4c e 4e); porém, na comparação entre o Método dos Perfis e o Método do Número de Richardson, houve um aumento, alcançando um ótimo índice de 0,97 (Figura 4f), ou seja, os valores de alturas da CLC encontrados nestes dois métodos apresentaram uma similaridade muito grande, com uma diferença de apenas $30 \mathrm{~m}$.

$$
\text { a) }
$$

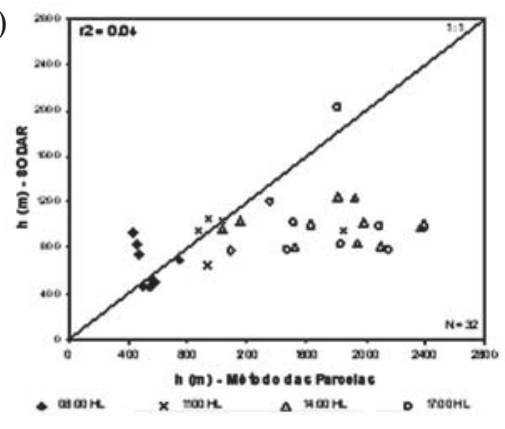

c)

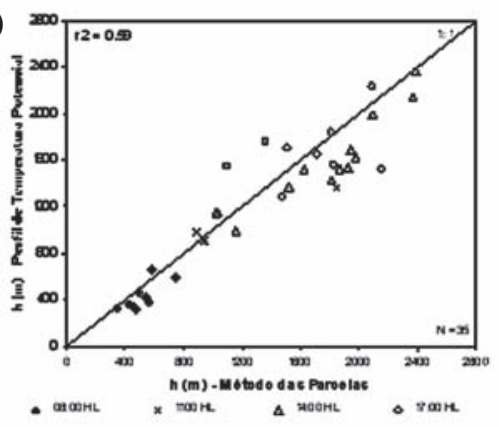

e)

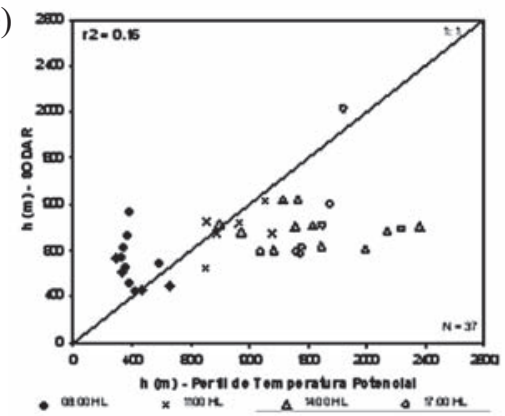

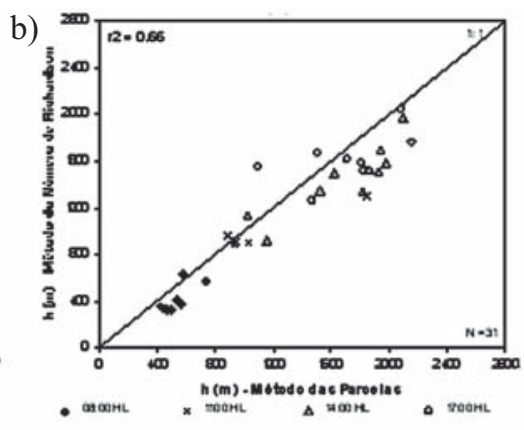

d)
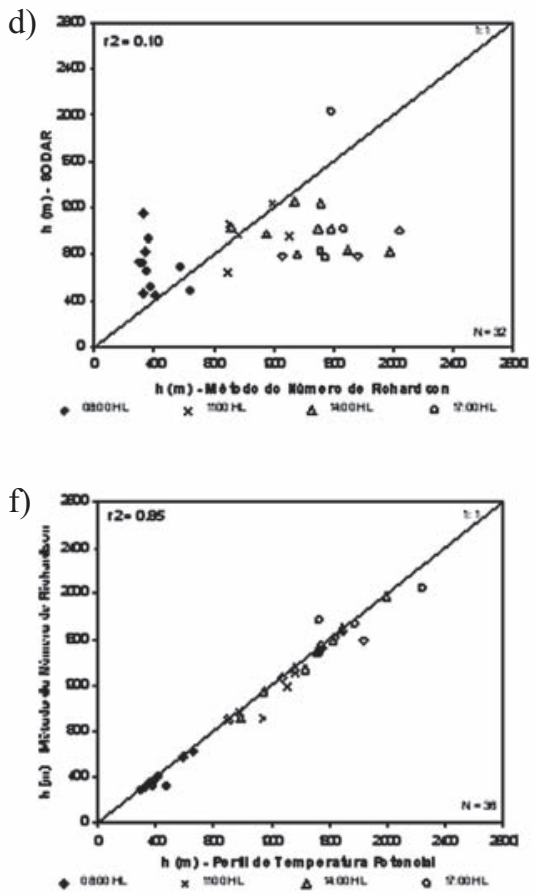

Figura 3 - Comparação entre os métodos de estimativa da altura da CLC durante a Fase I: (a) Método das Parcelas X SODAR; (b) Método das Parcelas X Método do Número de Richardson; (c) Método das Parcelas X Método dos Perfis; (d) SODAR X Método do Número de Richardson; (e) SODAR $\mathrm{X}$ Método dos Perfis; (f) Método do Número de Richardson X Método dos Perfis. Onde $\mathbf{N}$ corresponde ao número de casos correlacionados. 
a)

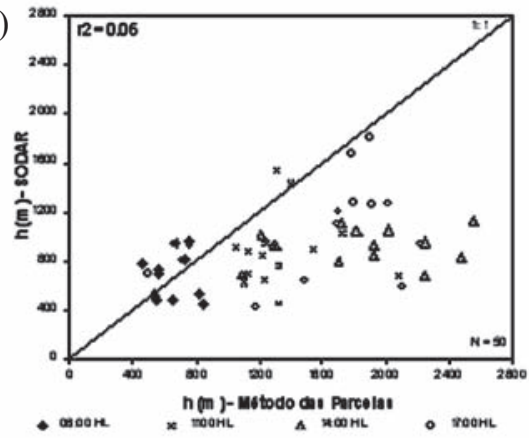

c)

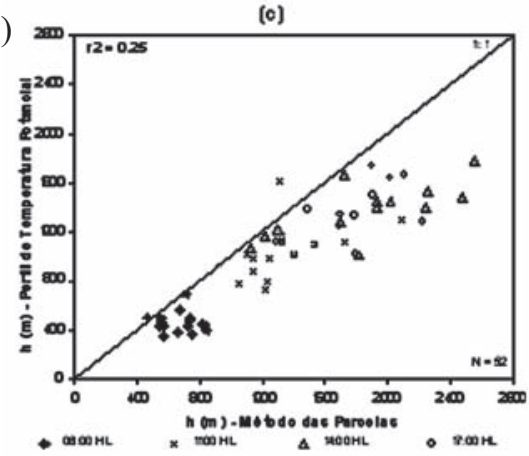

e)

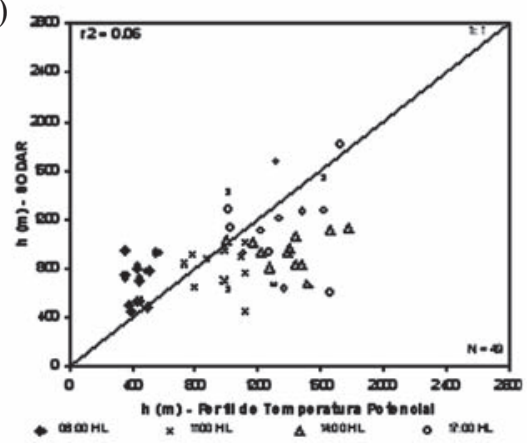

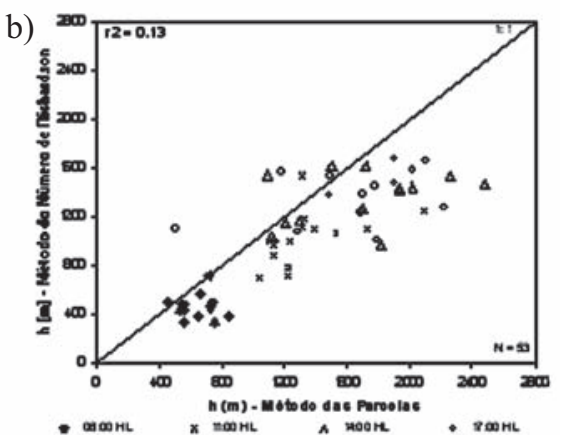

d)

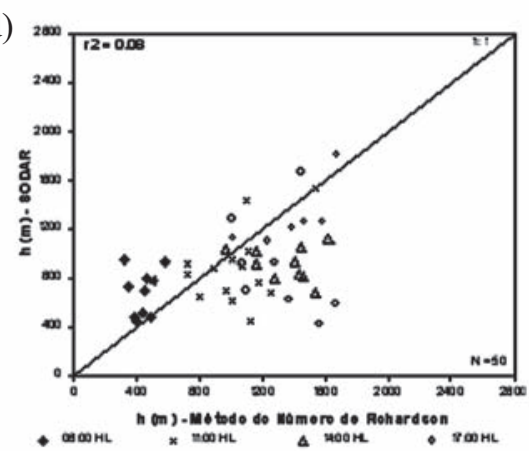

f)

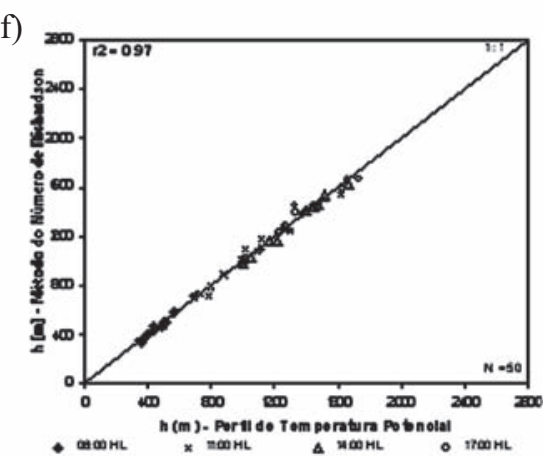

Figura 4 - Comparação entre os métodos de estimativa da altura da CLC durante a Fase II: (a) Método das Parcelas X SODAR; (b) Método das Parcelas X Método do Número de Richardson; (c) Método das Parcelas X Método dos Perfis; (d) SODAR X Método do Número de Richardson; (e) SODAR X Método dos Perfis; (f) Método do Número de Richardson X Método dos Perfis. Sendo que $N$ corresponde ao número de casos correlacionados.

\section{CONCLUSÕES}

Este trabalho teve como objetivo fazer uma comparação entre quatro diferentes métodos de estimativa da altura da CLC, utilizando dois métodos objetivos (Método do Número de Richardson e Método das Parcelas), um subjetivo (Método do Perfil) e um método de medição direta (SODAR).

Os resultados mostraram que o Método das Parcelas, em média, superestima as alturas encontradas em todos os outros métodos apresentando as melhores correlações com o Método do Número de Richardson $\left(\mathrm{r}^{2}=0,66\right)$ e o Método do Perfil $\left(\mathrm{r}^{2}\right.$ $=0,59)$, apenas para a Fase I, sendo que durante a Fase II as correlações encontradas com o Método das Parcelas foram baixas atingindo apenas 0,25 , quando comparada com o Método do Perfil. A superestimação deste método ocorre pelo fato de se considerar que a interseção do perfil de temperatura com a adiabática seca é um suposto ponto de equilíbrio do crescimento das parcelas de ar ou térmicas e que seja invariante com o tempo, o que não é verdade.

As alturas obtidas pelo SODAR foram as que obtiveram os menores valores de correlação quando comparadas com os outros métodos, permanecendo quase sempre abaixo dos outros valores, com exceção das medidas feitas às 08:00 HL, onde o aparelho mediu erroneamente a altura da CLR ao invés da CLC 
rasa. Outra explicação para os baixos valores de $\mathbf{h}$ é devido ao alcance médio do SODAR, que é de $1500 \mathrm{~m}$, e a partir desta altura é feita uma estimação de h, e para a área de estudo, onde a CLC comumente ultrapassa $1800 \mathrm{~m}$ durante a estação seca (Fisch, 1996), seria mais viável a utilização de um SODAR com alcance médio acima de $2000 \mathrm{~m}$.

Os métodos do Número de Richardson e o do Perfil, quando comparados entre si, obtiveram as melhores correlações, com valores de 0,85 e 0,97 tanto para a Fase I e Fase II, respectivamente, com pequenas diferenças (cerca de $30 \mathrm{~m}$ para a Fase II) nas alturas encontradas. O Método do Número de Richardson não é muito utilizado pelo fato de depender dos valores de $\theta$ e dos valores de velocidade do vento, o que torna-se mais complexa em relação ao Método do Perfil (que somente necessita da temperatura potencial). Pela simplicidade e efetividade, o Método do Perfil continua sendo o mais utilizado, além de ter a vantagem de se poder calcular $\mathbf{h}$, tanto pelo perfil de $\theta$ quanto pelo perfil de $\mathbf{q}$, caso haja algum problema com um dos sensores. Estes resultados demonstram a aplicabilidade do uso do Método dos Perfis para a estimativa da CLC, principalmente em modelos operacionais de Previsão Numérica de Tempo.

\section{REFERÊNCIAS BIBLIOGRÁFICAS}

ANDRADE, E. S. De, SA, L.D. de A., JORGE, M.P.P.M. Estimativa da altura da Camada Limite Atmosférica acima do Pantanal: estudo comparativo usando radiossondagem e SODAR. XIII Congresso Brasileiro de Meteorologia (CBMET), Fortaleza-CE, CD-ROM, Anais, Outubro de 2004.

BAUMANN, K. e PIRINGER, M. Two-years of boundary layer measurements with a sodar- statistics and applications. Physics and Chemistry of the Earth, Part B: Hydrology, Oceans and Atmosphere, v. 26, n. 3, p. 205-211, 2001.

BEYRICH, F. Mixing height estimation in the convective boundary layer using sodar data. Boundary Layer Meteorology, v. 74, n. 1, p. 1-18, 1995.

FISCH, G.; TÓTA J.; MACHADO, L.A.T.; SILVA DIAS, M.A.F.; LYRA, R. F. da F.; NOBRE, C. A.; DOLMAN, A. J.; GASH, J. H. C. The convective boundary layer over pasture and forest in Amazonia. Theoretical and Applied Climatology, v.78, n.1-3, p. 47-59, 2004.

G. Camada Limite Amazônica: Aspectos Observacionais e de Modelagem. 155 p. (INPE- 6123-TPT/584). Tese de Doutorado em Meteorologia, Instituto Nacional de Pesquisas Espaciais, São José dos Campos, 1996.
, CASTRO, L.C., GUEDES, R.L., QUINSAN JR., H.R. Uso do sistema GPS para a determinação do vento em altitude. Revista Brasileira de Meteorologia, v. 13, n. 1, p. $65-70,1998$.

HENNEMUTH, B.; LAMMERT, A. Determination of the atmospheric boundary layer height from radiosonde and lidar backscatter. Boundary Layer Meteorology, v.118. n.2, p. 242-262, 2006.

NOBRE, C. A.; SELLERS, P. J.; SHUKLA, J. Amazonian Deforestation and Regional Climate Change. Journal of Climate, v. 4, n. 10, p.957-988, 1991.

ROCHA, H. R. Modelagem da interação Biosfera-Atmosfera para a área de floresta e pastagem na Amazônia. 127 p. (INPE- 5454TPI/496). Dissertação de Mestrado em Meteorologia, INPE, São José dos Campos, 1992.

REMTECH. Doppler Sodar operating manual. Techinical report. DT94/003 - 09, 1994.

SANTOS, L. A. R. Análise e caracterização da camada limite convectiva em área de pastagem, durante o período de transição entre a estação seca e chuvosa na Amazônia (Experimento RaCCI-LBA). 118 p. (INPE- 551.5510.522). Dissertação de Mestrado em Meteorologia, INPE, São José dos Campos, 2005.

SEIBERT, P.; BEIRICH, F.; GRYNING, S.; JOFFRE, S.; RASMUSSEN,A.; TERCIER, P. Review and intercomparison of operational methods for the determination of the mixing height. Atmospheric Environment, v.34, n. 7, p.1001$1027,2000$.

STULL, R. B. An Introduction to Boundary Layer Meteorology. Dordrecht, Kluwer Academic Publishers, 666 p., 1988.

VOGELEZANG D. H. P.; HOLTSLAG A. A. M. Evaluation and model impacts of alternative boundary layer height fomulations. Boundary Layer Meteorology, v.81, n. 2, p.245-269, 1996. 